\title{
How to optimise duration of antibiotic treatment in patients with sepsis?
}

DOI:

10.1136/bmj.m4357

\section{Document Version}

Final published version

Link to publication record in Manchester Research Explorer

\section{Citation for published version (APA):}

Hellyer, T., Mantle, T., Mcmullan, R., \& Dark, P. (2020). How to optimise duration of antibiotic treatment in patients with sepsis? British Medical Journal, m4357. https://doi.org/10.1136/bmj.m4357

\section{Published in:}

British Medical Journal

\section{Citing this paper}

Please note that where the full-text provided on Manchester Research Explorer is the Author Accepted Manuscript or Proof version this may differ from the final Published version. If citing, it is advised that you check and use the publisher's definitive version.

\section{General rights}

Copyright and moral rights for the publications made accessible in the Research Explorer are retained by the authors and/or other copyright owners and it is a condition of accessing publications that users recognise and abide by the legal requirements associated with these rights.

\section{Takedown policy}

If you believe that this document breaches copyright please refer to the University of Manchester's Takedown Procedures [http://man.ac.uk/04Y6Bo] or contact uml.scholarlycommunications@manchester.ac.uk providing relevant details, so we can investigate your claim.

\section{OPEN ACCESS}


1 Newcastle University, Newcastle, UK

2 Manchester Medical School, Manchester, UK

3 Belfast Health \& Social Care Trust and Reader, Centre for Experimental Medicine, Queen's University Belfast. Belfast, UK

4 Manchester NIHR Biomedical Research Centre, University of Manchester, Manchester, UK

5 Northern Care Alliance NHS Group, Greater Manchester, UK

Correspondence to paul.m.dark@manchester.ac.uk Cite this as: BMJ2020;371:m4357 http://dx.doi.org/10.1136/bmj.m4357 Published: 23 November 2020

\section{UNCERTAINTIES}

\section{How to optimise duration of antibiotic treatment in patients with sepsis?}

\author{
TP Hellyer, ${ }^{1}$ T Mantle, ${ }^{2}$ R McMullan, ${ }^{3}$ P Dark ${ }^{4,5}$
}

What you need to know

- Guidelines recommend 7 to 10 days of antibiotic treatment for patients with sepsis, but a shorter duration may be safe in certain individuals

- Low quality evidence suggests that monitoring biomarkers such as procalcitonin can reduce antibiotic duration in sepsis by about one day, but the effectiveness in severe disease and in low resource settings is not known

- Use your clinical judgement to consider the diagnostic evidence, signs of resolution or worsening of infection, and individual risk when making a decision about the choice and duration of antibiotic treatment

Sepsis occurs when the body's response to infection is imbalanced. It can result in life threatening organ dysfunction. ${ }^{1}$ About 49 million patients had sepsis worldwide in 2017 and 11 million people died of the condition. ${ }^{2}$ Among those hospitalised with sepsis, $17 \%$ die in hospital and a further $15 \%$ die within a year of hospital discharge. ${ }^{34}$

Antibiotics represent the cornerstone of treatment. The Surviving Sepsis Campaign (SSC) consensus guidelines recommend treatment for 7 to 10 days, but this is a weak recommendation. ${ }^{5}$ Use of antibiotics risks the emergence of antimicrobial resistance and antibiotic-associated infections, such as Clostridiodes difficile. Patients may suffer from toxic effects of the drugs. ${ }^{6}$ Balancing the need to treat severe infections effectively against the risks of overuse of antibiotics is central to the principle of antibiotic stewardship. For severe covid-19, antibiotic stewardship remains important for critically ill patients with pneumonitis and sepsis who are commonly treated with broad spectrum antibiotics. ${ }^{7}$ Antibiotic overuse in sepsis results in substantial risk of acquiring difficult-to-treat infections, with further risk of sepsis and poor patient outcomes. 5

The optimum duration of antibiotic treatment for patients with sepsis is uncertain. A biomarker-guided approach-for example, using serial measurements of procalcitonin to determine optimal duration of antibiotics-has been studied. Trials have evaluated fixed short periods of antibiotic treatment in patients with specific infections that can cause sepsis. It is uncertain whether any of these approaches can safely guide decisions in practice on the duration of antibiotic treatment for patients with sepsis.

\section{Sources and search selection}

We searched Medline and Embase from inception to 16 October 2019. We searched for randomised controlled trials and systematic reviews of biomarker-guided or fixed durations of antibiotic treatment compared with standard care for adult patients with sepsis. We used terms such as "sepsis", "bacterial infections", "bacteraemia", "anti-bacterial agents", "protein precursors", "duration", and variations on these terms. We also included studies that investigated specific infections that commonly cause sepsis. The three commonest infection sources of sepsis are respiratory tract, urinary tract, and intra-abdominal infections, as found in the National Confidential Enquiry into Patient Outcome and Death 2015 Sepsis report. Twenty eight randomised controlled trials and 29 systematic reviews or meta-analyses were considered (supplementary files 1,2). References were checked for other relevant articles not retrieved in the search. Two further randomised controlled trial studies were published in 2020 (supplementary files, table 1). To provide a concise narrative review, we selected up-to-date meta-analyses and key trials that addressed antibiotic treatment duration in sepsis and in major infections that cause sepsis (tables 1, 2, supplementary files).

\section{What is the evidence of uncertainty?}

\section{Biomarker-guided antibiotic treatment}

Several biomarkers have been investigated in sepsis, but our search only identified procalcitonin and $\mathrm{C}$ reactive protein as biomarkers used to alter duration of antibiotic treatment. High quality evidence is lacking to recommend the routine use of procalcitonin-guided antibiotic treatment decisions for adult patients with sepsis. ${ }^{8}$ Table 1

(supplementary files) provides a summary of the evidence. Studies do not report longer-term patient outcomes from sepsis, and evidence for the cost-effectiveness of biomarker-guided antibiotic treatment duration is lacking. ${ }^{8}$

Measuring circulating procalcitonin is seen to reduce antibiotic duration by about one day ( 9.3 versus 10.4 days) ${ }^{8-10}$ and results in a lower 30 day mortality (odds ratio $0.89,95 \%$ confidence interval 0.80 to 0.99 ) in patients with sepsis and those with respiratory tract infections, as per meta-analyses. ${ }^{910}$ These durations are not particularly short and reductions in duration were not observed in the sickest patients. The quality of evidence is low, as most trials do not provide details on standards of care and are at high risk of bias. ${ }^{8} 9$ Trials had different algorithms, which used daily or intermittent procalcitonin measurement, and one trial had a single measurement. ${ }^{9}$

One trial (97 patients) in Brazil evaluated $C$ reactive protein in patients with suspected or confirmed sepsis in intensive care units and found no difference in antibiotic duration between approaches guided by procalcitonin or $\mathrm{C}$ reactive protein. ${ }^{11}$ 
No trials have been completed in the UK and few trials have been conducted in low and middle income countries. Most patients with sepsis live in low and middle income countries. ${ }^{212}$ It is uncertain whether these findings can be realised in all healthcare systems, particularly in low resource settings.

\section{Fixed short duration of antibiotic treatment}

Respiratory tract infections, urinary tract infections, and intra-abdominal infections are the commonest infection sources of sepsis. ${ }^{13}$ The duration of antibiotic treatment differs depending on the source of infection. ${ }^{14}$ Evidence from trials suggests that five days of antibiotics for community acquired pneumonia, ${ }^{15}$ seven days or fewer for urinary tract infections, ${ }^{16} 17$ and four days for intra-abdominal infections with source control ${ }^{18}$ have effectiveness comparable with longer duration of antibiotics over 10-14 days. Patients who require critical care support are generally excluded from these trials. High quality evidence is lacking for high severity and high risk groups, where longer durations may be required until clinical stability is reached. ${ }^{19}$

\section{Is ongoing research likely to provide relevant evidence?}

We searched Clinicaltrials.gov and the ISRCTN registry and identified five ongoing trials (box 1). Three trials compare fixed duration treatments and will add to the evidence on the effectiveness and safety of short term antibiotic duration. One trial is investigating whether a diagnostic bundle could reduce antibiotic use safely. We are conducting a double-blinded trial (ADAPT-Sepsis) that will provide evidence on the clinical and cost effectiveness of biomarker-guided antibiotic duration in UK healthcare settings, and which has recently been extended to include patients with covid-19 and sepsis as part of the UK's portfolio of urgent public health research during the pandemic. Evidence on long term clinical outcomes is lacking. The ADAPT-Sepsis trial will help address this using linked national healthcare digital patient records, and the $\mathrm{SAB} 7$ trial will address this with in-trial follow-up until 6 months.

\section{Box 1: Ongoing trials on optimising antibiotic treatment in patients with sepsis}

- ADAPT-Sepsis will determine the utility of $\mathrm{C}$ reactive protein and procalcitonin to reduce antibiotic use in patients with suspected sepsis in UK critical care units. ISRCTN47473244.

- The BALANCE trial will determine whether seven days of adequate antibiotic treatment is non-inferior to 14 days of treatment for critically ill patients with bacteraemia. NCTo3005145.

- The SAB7 trial will determine whether seven days of antibiotics is non-inferior to 14 days of antibiotics for patients with uncomplicated Staphylococcus aureus bacteraemia. NCT03514446.

- A trial comparing five days with 10 days of antibiotic treatment for patients with presumed infections. NCTo2899143.

- The PIBCAP trial will compare the utility of a pneumonia investigation bundle, including multiplex real-time polymerase chain reaction assays, with investigation and treatment as per guidelines from the National Institution for Health and Care Excellence. Primary outcome will assess clinical response and antibiotic use (defined daily dose). ISRCTN42850134.

\section{What should we do in light of the uncertainty?}

Antibiotic treatment duration could be safely shortened for patients with sepsis, but other factors must be considered, including the site of infection and severity. For example, the SSC guidelines state that shorter antibiotic courses may be appropriate for patients with rapid clinical resolution following effective source control of intra-abdominal or urinary sepsis and those with anatomically uncomplicated pyelonephritis (weak recommendations, low quality of evidence). ${ }^{5}$ Control of the source of infection (by surgical or radiological means) is important, particularly for intra-abdominal infections. ${ }^{18}$ Some pathogens, such as non-fermenting Gram-negative bacilli, may require prolonged antibiotic treatment to avoid relapse of infection. ${ }^{20}$

Patients with sepsis have multiple morbidities and require complex care. Other uncertainties pertaining to antibiotic treatment in sepsis include choice of antibiotic, dosing, starting and stopping treatment, and considerations in patients with complications such as shock or organ failure. The clinical multidisciplinary team must weigh up diagnostic evidence, signs of resolution or worsening of infection, and individual risk in the context of patient choice. We suggest following the principles of antibiotic stewardship in the context of clinical judgement by identifying and controlling the infectious source, as well as narrowing, and subsequently discontinuing, antibiotics as soon as laboratory, radiological, and clinical parameters permit.

\section{Education into practice}

- What factors do you consider when deciding the duration of antibiotic treatment in a patient?

- How do you use laboratory results from microbiology sampling, biomarkers, and routine blood tests in your initial suspicion of infection or to alter decisions on antibiotic duration?

- When delivering urgent empirical antibiotics for sepsis, what is your antibiotic stewardship plan?

\section{Recommendations for future research}

Future research should determine the optimal duration of antibiotic treatment in patients in whom antibiotic exposure can be prolonged owing to severe disease, and in those who require organ support.

- Population: Patients with sepsis who require critical care organ support

- Intervention: Short duration antibiotics

- Comparator: Standard practice

- Non-inferior clinical outcomes: Antibiotic use (duration, defined daily dose), mortality, duration of organ support

\section{What patients need to know}

- Sepsis is a life threatening condition and appropriate antibiotics are needed urgently to treat infection. Guidelines recommend 7 to 10 days of antibiotics but the evidence for this is of low quality

- Optimising the duration of antibiotic treatment is important to reduce harms such as antibiotic resistance and antibiotic associated infections

- Measuring certain biomarkers in blood has been shown safely to reduce duration of antibiotic treatment by about a day, but this evidence is of low quality. Fixed short duration of antibiotics is also shown to be safe in certain infections that can lead to sepsis

- We don't know if these approaches are safe in patients with severe disease or those requiring intensive care, and in low resource settings

- Ongoing research aims at delivering shorter, safer courses of antibiotic treatment for patients with sepsis

\section{How patients were involved in the creation of this article}

Patients were not directly involved in the writing of this article. We are conducting the ADAPT-Sepsis trial and our study team includes members of the Intensive Care Society's Patients and Relatives group and the 
Manchester NIHR Biomedical Research Centre's Patient and Public Involvement and Engagement group. We are engaging with these groups to guide the design, delivery, and communication of our research.

Competing interests: Tom Hellyer is supported by the National Institute for Health Research (NIHR) as an academic clinical lecturer and Paul Dark is supported by the Manchester NIHR Biomedical Research Centre. Paul Dark is on the clinical advisory board of DNA Electronics delegated by Salford Royal NHS Trust to advise on clinical applications and safety of rapid diagnostic technology for bloodstream infections. Ronan McMullan is involved in research with Randox Ltd for a point-of-care test in sepsis funded by a grant from Innovate UK. He has given lectures on fungal infections at educational meetings sponsored by Gilead.

Provenance and peer review: commissioned; externally peer reviewed.

1 Singer M, Deutschman CS, Seymour CW, etal. The third international consensus definitions for sepsis and septic shock (sepsis-3). JAMA 2016;315:801-10. doi: 10.1001/jama.2016.0287 pmid: 26903338

2 Rudd KE, Johnson SC, Agesa KM, etal. Global, regional, and national sepsis incidence and mortality, 1990-2017: analysis for the Global Burden of Disease Study. Lancet 2020;395:200-11. doi: 10.1016/S0140-6736(19)32989-7 pmid: 31954465

3 Fleischmann C, Scherag A, Adhikari NKJ, etallnternational Forum of Acute Care Trialists. Assessment of global incidence and mortality of hospital-treated sepsis. Current estimates and limitations. Am J Respir Crit Care Med 2016;193:259-72. doi: 10.1164/rccm.201504-07810C pmid: 26414292

4 Shankar-Hari M, Harrison DA, Ferrando-Vivas P, Rubenfeld GD, Rowan K. Risk factors at index hospitalization associated with longer-term mortality in adult sepsis survivors. JAMA Netw Open 2019;2:e194900. doi: 10.1001/jamanetworkopen.2019.4900 pmid: 31150081

5 Rhodes A, Evans LE, Alhazzani W, etal. Surviving sepsis campaign: international guidelines for management of sepsis and septic shock: 2016. Crit Care Med 2017;45:486-552. doi: 10.1097/CCM.0000000000002255 pmid: 28098591

6 Klompas M, Calandra T, Singer M. Antibiotics for sepsis-finding the equilibrium. JAMA 2018;320:1433-4. doi: 10.1001/jama.2018.12179 pmid: 30242350

7 Seaton RA, Gibbons CL, Cooper L, et al. Survey of antibiotic and antifungal prescriptions in patients with suspected and confirmed COVID-19 in Scottish hospitals. I Infect 2020 https://www.ncbi.nlm.nih.gov/pmc/articles/PMC7518971/

8 Westwood M, Ramaekers B, Whiting P, etal. Procalcitonin testing to guide antibiotic therapy for the treatment of sepsis in intensive care settings and for suspected bacterial infection in emergency department settings: a systematic review and cost-effectiveness analysis. Health Technol Assess 2015;19:1-236. doi: 10.3310/hta19960 pmid: 26569153

9 Wirz Y, Meier MA, Bouadma L, etal. Effect of procalcitonin-guided antibiotic treatment on clinical outcomes in intensive care unit patients with infection and sepsis patients: a patient-level meta-analysis of randomized trials. Crit Care 2018;22:191. doi: 10.1186/s13054-018-2125-7 pmid: 30111341

10 Schuetz P, Wirz Y, Sager R, etal. Effect of procalcitonin-guided antibiotic treatment on mortality in acute respiratory infections: a patient level meta-analysis. Lancet Infect Dis 2018;18:95-107. doi: 10.1016/S1473-3099(17)30592-3 pmid: 29037960

11 Oliveira CF, Botoni FA, Oliveira CRA, etal. Procalcitonin versus C-reactive protein for guiding antibiotic therapy in sepsis: a randomized trial. Crit Care Med 2013;41:2336-43. doi: 10.1097/CCM.0b013e31828e969f pmid: 23921272

12 Rudd KE, Kissoon N, Limmathurotsakul D, etal. The global burden of sepsis: barriers and potential solutions. Crit Care 2018;22:232. doi: 10.1186/s13054-018-2157-z pmid: 30243300

13 Goodwin APL, Srivastava V, Shotton H, Protopapa K, Butt A, Mason M. National Confidential Enquiry into Patient Outcome and Death. Just Say Sepsis! A review of the process of care received by patients with sepsis. A report by the National Confidential Enquiry into Patient Outcome and Death, November 2015. 2015. https://www.ncepod.org.uk/2015report2/downloads/JustSaySepsis_FullReport.pdf

14 Bouadma L, Luyt C-E, Tubach F, etalPRORATA trial group. Use of procalcitonin to reduce patients' exposure to antibiotics in intensive care units (PRORATA trial): a multicentre randomised controlled trial. Lancet 2010;375:463-74. doi: 10.1016/50140-6736(09)61879-1 pmid: 20097417

15 Uranga A, España PP, Bilbao A, etal. Duration of antibiotic treatment in community-acquired pneumonia. JAMA Intern Med 2016;176:1257-65. doi: 10.1001/jamainternmed.2016.3633 pmid: 27455166

16 Eliakim-Raz N, Yahav D, Paul M, Leibovici L. Duration of antibiotic treatment for acute pyelonephritis and septic urinary tract infection -7 days or less versus longer treatment: systematic review and meta-analysis of randomized controlled trials. J Antimicrob Chemother 2013;68:2183-91. doi: 10.1093/jac/dkt177 pmid: 23696620

17 Yahav D, Franceschini E, Koppel F, etalBacteremia Duration Study Group. Seven versus 14 days of antibiotic therapy for uncomplicated gram-negative bacteremia: a noninferiority randomized controlled trial. Clin Infect Dis 2019;69:1091-8. doi: 10.1093/cid/ciy1054 pmid: 30535100

18 Sawyer RG, Claridge JA, Nathens AB, etalSTOP-IT Trial Investigators. Trial of short-course antimicrobial therapy for intraabdominal infection. N Engl J Med 2015;372:1996-2005. doi: 10.1056/NEJMoa1411162 pmid: 25992746

19 National Institute for Health and Care Excellence. Pneumonia (community-acquired): antimicrobial prescribing NICE guideline (NG 138). NICE Guideline 2019. https://www.nice.org.uk/guidance/ng138
20 Chastre J, Wolff M, Fagon J-Y, etalPneumA Trial Group. Comparison of 8 vs 15 days of antibiotic therapy for ventilator-associated pneumonia in adults: a randomized trial. JAMA 2003:290:2588-98. doi: 10.1001/jama.290.19.2588 pmid: 14625336 